\title{
Environmental Change and Nonlinearity - A Summary of a new Cross-cutting and Integrative Initiative within Focus 3
}

\section{Forcing-Response Mechanisms and Focus 3 Activities}

A key IGBP objective is to understand the relationships between forcings, by human actions and climate variability, and responses by processes within the Earth system. In contemporary process studies, there is often the opportunity to make direct measurement of these relationships (or transfer functions). Thus for a variety of modern impacts, such as the effects of deforestation or extreme precipitation events on soil erosion, there are data available that may be used to produce transfer functions for input into predictive process models. Process studies often show that the transfer functions are nonlinear - a forcing input is disproportionately related to the output response, usually because of the mediating effects of thresholds and feedbacks. The Focus 3 Activities, examining sedimentary archival records of change in fluvial environments (LUCIFS), aquatic environments (LIMPACS) and the terrestrial environment (HITE), together represent the natural methodological extension of contemporary process studies that are constrained by short timescales. Bridging the gap between studies of modern processes and paleo-processes is now a major challenge for the PAGES community and linked core projects, not least where the objective is to reconstruct the nonlinear nature of past or long term environmental change.

\section{Sediments and System Dynamics}

Focus 3 Activities utilize cascading systems as their major frameworks for study. Such systems normally provide delimited, and usually coupled, spatial entities (lake, floodplain and catchment) in which synergistic and independent impacts of human actions and climate on a wide range of ecological, biogeochemical, hydrological and geomorphological processes may be recorded in a sedimentary archive. Focusing on a sedimentary archive as a sequence of outputs from an aquatic, fluvial or terrestrial system highlights a need to appreciate that the behavior of a whole system may be different from the additive behavior of component parts - the 'reductionism versus holism' debate that brings into play a variety of theories arising from the mathematical treatment of model systems over the past few decades. There are extremely important implications for our explanation

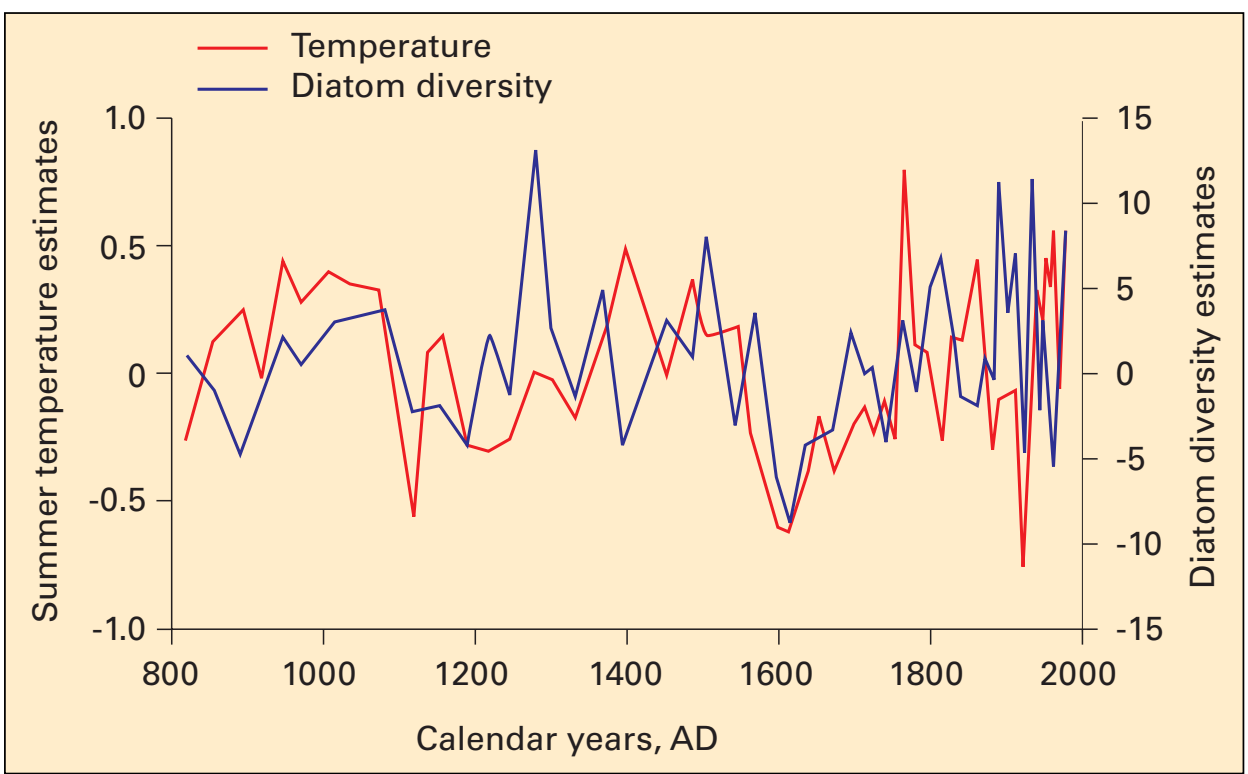

Figure 1: Northern Scandinavian tree-ring inferred summer temperature variations plotted together with diatom species richness estimates at Kassjön (northern Sweden) since AD 861 determined using rarefaction. The diatom species richness is shown with a lag of 20 years relative to the temperature data. Diatom richness is probably controlled by temperature changes driving biogeochemical processes in the catchment with a time lag of 20-25 years. Anderson et al. 1996. and understanding of forcing-response mechanisms if it can be demonstrated that changes in reconstructed environments are best described by nonlinear system behaviors. For example, our preconceptions of forcing-response mechanisms may require modification; the effects of perturbations may be highly dependent on spatial scale; the successful application of paleoecological transfer functions may be conditioned by system states; and some types of environmental change may be essentially unpredictable.

\section{Mechanisms of Environmental Change}

A classification of mechanisms which drive environmental change is problematic because they are likely to coexist, often synergistically, and vary across different temporal and spatial scales. But three categories serve to illustrate common concepts of change. First, extrinsic apparently linear mechanisms exist where responses are proportional to outputs and where relatively small intrinsic thresholds give rise to short or unmeasurable time-lags. Second, extrinsic nonlinear mechanisms exist where responses are disproportionate to forcings and where there may be long or measurable time lags because of significant intrinsic thresholds and feedback within the system (Figure 1). A component of these mechanisms may be deterministic chaos, where small differences in initial conditions determine the responses to a particular magnitude of forcing. Third, intrinsic 'nonlinear' mechanisms exist where systems display non-random changes which are independent of external forcings. Evidence for emergent complexity and selforganized criticality, two of the terms from systems theory which apply to this category, has been found in a recent study of laminated sediments at Holzmaar, Germany. Frequency distributions of sediment accumulation rate data (Figure 2) suggest that the catchment's sediment system in the midHolocene, before major human impact, may have reached the state of selforganized criticality. In this state, the magnitude of sediment loss from the catchment may have been extremely 
sensitive to large external perturbations but, paradoxically, variations recorded in the lake sediments may have also been completely independent of climatic or any other external forcing events. In many lake sediment records, trends in proxy records of processes may be interpreted as trajectories in system behavior driven by internal organizational mechanisms that lead to greater or lesser system complexity and new steady states (Figure 3). Identifying a system's dynamic state allows the transfer of information from systems theory, which provides a strong basis to develop new hypotheses about the controls on change and to gauge the extent to which predictive models may be successfully applied in similar modern systems.

\section{An Integrating Initiative}

The Focus 3 Steering Group have proposed that there should be a cross-cutting and integrating initiative with the general aim of promoting the importance of system dynamics and nonlinearity in forcing-response mechanisms within past environments. An

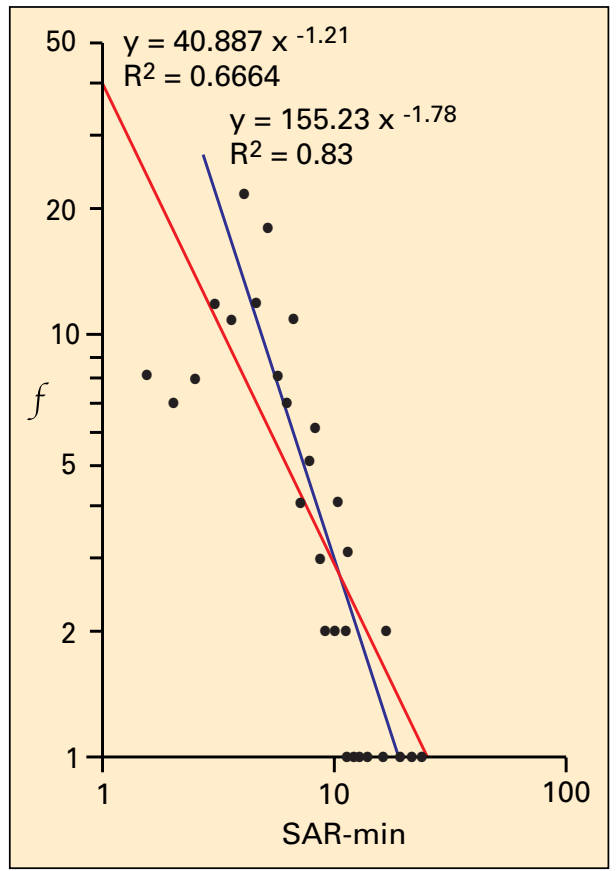

Figure 2: Frequency (f) distribution of minerogenic sediment accumulation rate (SAR-min) for the period 3000-10,000 cal. yr. $B P$ at Holzmaar, Germany, showing inverse power functions for the complete (red) and linear (blue) parts of the dataset with exponents lying between 0 and -2, indicative of nonlinear self-organized criticality. Dearing and Zolitschka (in press).

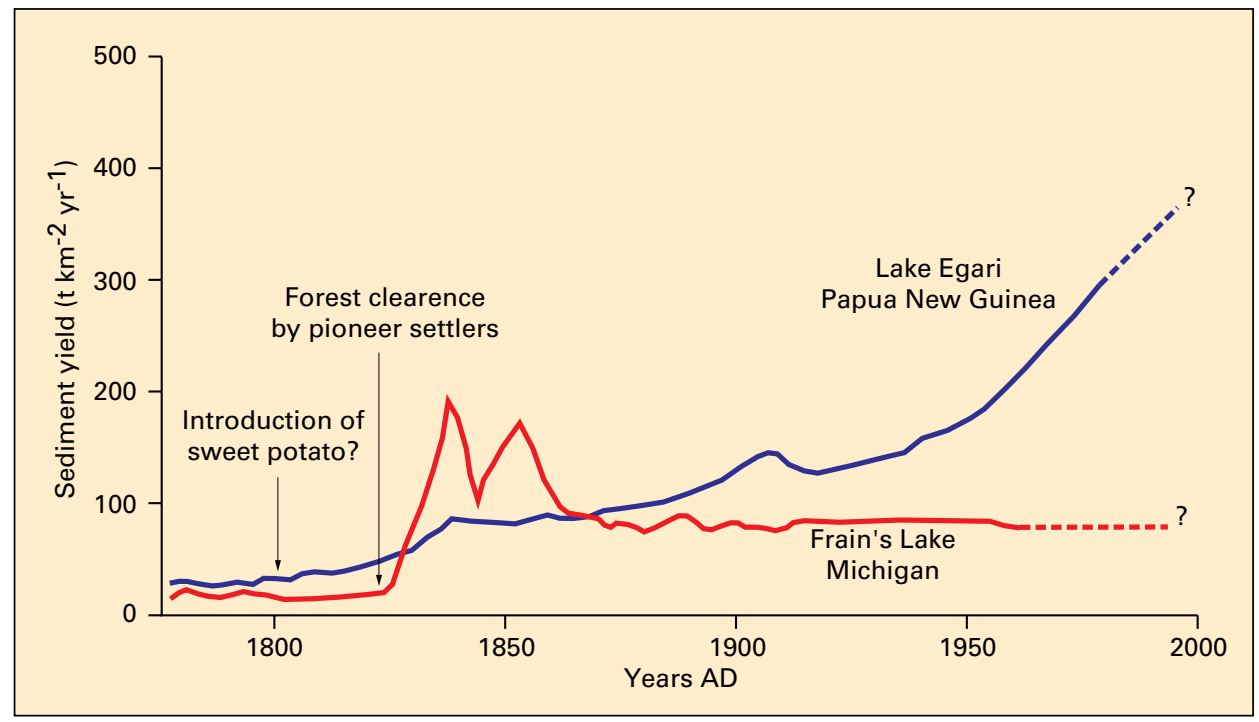

Figure 3: Reconstructed sediment yields since 1750 AD at Frain's Lake, Michigan, USA, (Davis 1976) and Lake Egari, Papua New Guinean Highlands (Oldfield et al., 1980), based on multiple core correlations and dating by pollen influx, historical records and ${ }^{210} \mathrm{~Pb}$. The curves show very different nonlinear responses to deforestation and land management, including a relatively rapid sequence of process response time - recovery — new steady state (Frain's Lake), and a long-term trajectory of increasing erosion possibly driven by progressive weakening of intrinsic erosion thresholds caused by a combination of local forest clearing and subsistence gardening (Lake Egari).

initial list of issues for consideration includes the following:

- developing and maintaining strong linkages between the three Focus 3 Activities in order to maximize our understanding of process operation in whole environments at a range of temporal and spatial scales

- designing methodologies that maximize the opportunities to identify system states and thresholds, perhaps by focusing on 'generic' environmental impacts (e.g. deforestation on biogeochemical processes) and gradients of spatial scale (e.g. nested lake-catchment sites)

- giving equal attention to records of responses and forcings, through site selection (e.g. sites with long and continuous historical or monitored records) and the development of stronger links between paleoecologists and environmental historians

- further application of high resolution and multi-proxy studies across periods of significant environmental change through the use of laminated sediments, ultra-fine scale sampling and the development of sensitive analytical techniques
- application of mathematical and statistical tools which identify system dynamics to existing and new time-series, with stronger collaborative links between mathematicians and paleoecologists

In the coming months, it is planned to produce a document for wide distribution that expands upon this summary. The PAGES and, particularly, Focus 3 communities are now invited to offer views on this proposal. Any individuals within Focus 3 who would like to become actively involved in this cross-cutting initiative, either as experts in specific methodological or mathematical fields, as discussants of strategy or as promoters of the aims within their Activity, should contact the author or the PAGES Project Office.

\section{John Dearing}

Department of Geography, University of Liverpool,UK j.dearing@liv.ac.uk

This article is based on a presentation given at the IGBP Congress, Japan, May 1999.

References for this article can be found at http://www.pages.unibe.ch/publications/ newsletters/ref992.html 